\title{
Precision Charmonium Spectroscopy From Lattice QCD
}

\author{
C. T. H. Davies, ${ }^{a}$ K. Hornbostel,${ }^{b}$ G. P. Lepage ${ }^{c}$ \\ A. J. Lidsey, ${ }^{a}$ J. Shigemitsu, ${ }^{d}$ J. Sloan ${ }^{e}$ \\ ${ }^{a}$ University of Glasgow, Glasgow, UK G12 8QQ. UKQCD collaboration. \\ ${ }^{b}$ Southern Methodist University, Dallas, TX 75275. \\ ${ }^{c}$ Newman Laboratory of Nuclear Studies, Cornell University, Ithaca, NY 14853. \\ ${ }^{d}$ The Ohio State University, Columbus, OH 43210. \\ ${ }^{e}$ Florida State University, SCRI, Tallahassee, FL 32306.
}

August 21, 2019

\begin{abstract}
We present results for Charmonium spectroscopy using Non-Relativistic QCD (NRQCD). For the NRQCD action the leading order spin-dependent and next to leading order spin-independent interactions have been included with tadpole-improved coefficients. We use multi-exponential fits to multiple correlation functions to extract ground and excited $S$ states. Splittings between the lowest $S, P$ and $D$ states are given and we have accurate values for the $S$ state hyperfine splitting and the $\chi_{c}$ fine structure. Agreement with experiment is good - the remaining systematic errors are discussed.
\end{abstract}

PACS numbers: 12.38.Gc, 14.40.Gx, 14.65.Dw, 12.39.Hg

\section{Introduction}

The study of heavy-heavy mesons is important for Lattice Gauge Theory not only because of the availability of experimental data for comparison but also because such systems allow a quantitative study of systematic errors which arise in lattice simulations at present. To study heavy-heavy mesons we use Non-Relativistic QCD (NRQCD) [1, 2] and previously we have reported a very successful study of the Bottomonium system[3]. This allowed the extraction of two fundamental parameters in QCD [4], the b-quark mass [5] and the strong coupling constant $\alpha_{s}$ [6]. Here we report on a similar study of the Charmonium spectrum. 
The starting point of NRQCD is to expand the original QCD lagrangian in powers of $v^{2}$, the typical quark velocity in a bound state. For the $J / \Psi$ system $v^{2} \sim 0.3$. Thus we systematically include relativistic errors order by order in $v^{2}$ away from a Non-Relativistic limit. Our action is the same one as used in [3] where relativistic corrections $\mathcal{O}\left(M v^{4}\right)$ have been included. This means that systematic errors from relativistic corrections will be $\mathcal{O}\left(M_{c} v^{6}\right)(=\approx 30-40 \mathrm{MeV})$ for the $J / \Psi$ system i.e. $10 \%$ in spin-independent splittings and $30 \%$ in spin-dependent splittings. This is considerably less accurate than for the $\Upsilon$ case [3] because $v^{2}$ is about a factor of 3 larger here. Other sources of systematic error include discretisation errors and errors from the absence of virtual quark loops because we use quenched configurations generated with the standard plaquette action. Finite volume errors should be negligible because of the relatively small size of the $J / \Psi$ system.

Shown in Figures (11) and (2) is the spectrum for Charmonium using Lattice NRQCD. The spectrum was calculated using an ensemble of 273 gauge field configurations generated with the standard Wilson action at $\beta=5.7$ [7]. To set the scale we fix our simulation result for the spin-averaged $1 \mathrm{P}-1 \mathrm{~S}$ splitting to its experimental value of $458 \mathrm{MeV}$. This gives $a^{-1}=1.23(4) \mathrm{GeV}$, where the uncertainty is purely statistical. Since we are working in the quenched approximation this value can be and is different both from that obtained at the same value of $\beta$ using light hadron spectroscopy [8] or using Upsilon spectroscopy [9]. We expect a value fixed from heavyonium to be more accurate than that from light hadron spectroscopy because spin-independent splittings in the heavy quark sector are independent of quark mass to a good approximation and systematic errors are under better control [1].

To fix the bare quark mass in the action, $M_{c}^{0}$, we plot a dispersion relation correct up to $\mathcal{O}\left(v^{4}\right)$ for the $\eta_{c} . M_{c}^{0}$ is then tuned until the simulation value for the kinetic mass is equal to the experimental value of the mass of the $\eta_{c}(2.98$ $\mathrm{GeV})$. We find that using $a M_{c}^{0}=0.8$ gives $M\left(\eta_{c}\right)=3.0(1) \mathrm{GeV}$ with $a^{-1}=1.23(4)$ $\mathrm{GeV}$.

In Figure (11) the whole Charmonium spectrum is shown and in Figure (2) the spin-dependent splittings are shown in more detail. In Figure (2) it can be seen that although the general pattern of splittings for the $\mathrm{S}$ and $\mathrm{P}$ states is reproduced well, systematic errors are visible above the statistical errors. It should then be possible in the future to observe systematic improvements to the current calculation, when higher order relativistic corrections are included and further discretisation and quenching errors are removed.

We give details in section 2 of our evolution equation and the quark Greens function used to make up meson correlation functions. Section 3 describes the results from the simulation using multi-exponential fits. We illustrate the need for multiple smearing functions to obtain smaller statistical errors. Section 4 compares simulation results to experiment and section 5 contains our conclusion. 


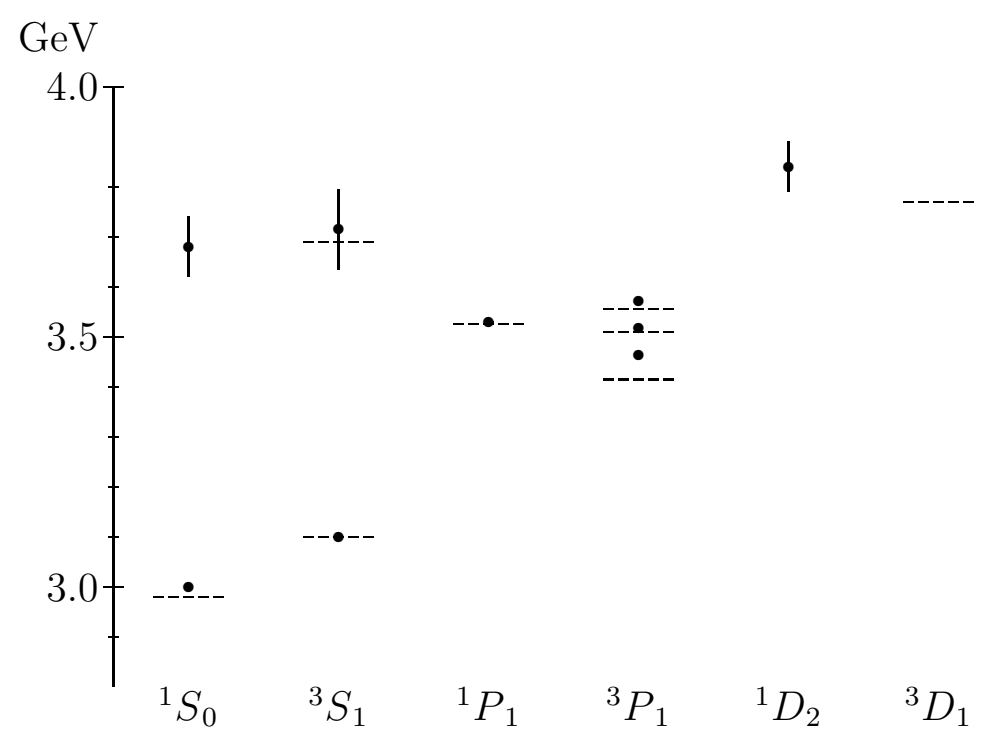

Figure 1: NRQCD simulation results for the spectrum of the $J / \Psi$ system using an inverse lattice spacing of $1.23 \mathrm{GeV}$, fixed from the spin-averaged $1 \mathrm{P}-1 \mathrm{~S}$ splitting. The ${ }^{1} S_{0}$ mass is fixed at $3.0 \mathrm{GeV}$, from a fit to the kinetic mass. Experimental values are indicated by dashed lines. Error bars are shown where visible, and only indicate statistical uncertainties.

\section{Evolution Equation and Quark propagators}

One of the advantages of the formulation of NRQCD is that it involves a simple difference equation in the temporal direction. This allows the evolution of the quark Green function as an initial value problem which can be solved with one sweep through the lattice. We define our quark Green function to be initially

$$
G_{1}=\left(1-\frac{a H_{0}}{2 n}\right)^{n} U_{4}^{\dagger}\left(1-\frac{a H_{0}}{2 n}\right)^{n} \delta_{\vec{x}, 0}
$$

and then continue to evolve using

$$
G_{t+1}=\left(1-\frac{a H_{0}}{2 n}\right)^{n} U_{4}^{\dagger}\left(1-\frac{a H_{0}}{2 n}\right)^{n}(1-a \delta H) G_{t} \quad(t>1) .
$$

On the lattice, the kinetic energy operator is

$$
H_{0}=-\frac{\Delta^{(2)}}{2 M_{c}^{0}}
$$

and the correction terms are

$$
\delta H=-c_{1} \frac{\left(\Delta^{(2)}\right)^{2}}{8\left(M_{c}^{0}\right)^{3}}+c_{2} \frac{i g}{8\left(M_{c}^{0}\right)^{2}}(\boldsymbol{\Delta} \cdot \mathbf{E}-\mathbf{E} \cdot \boldsymbol{\Delta})
$$




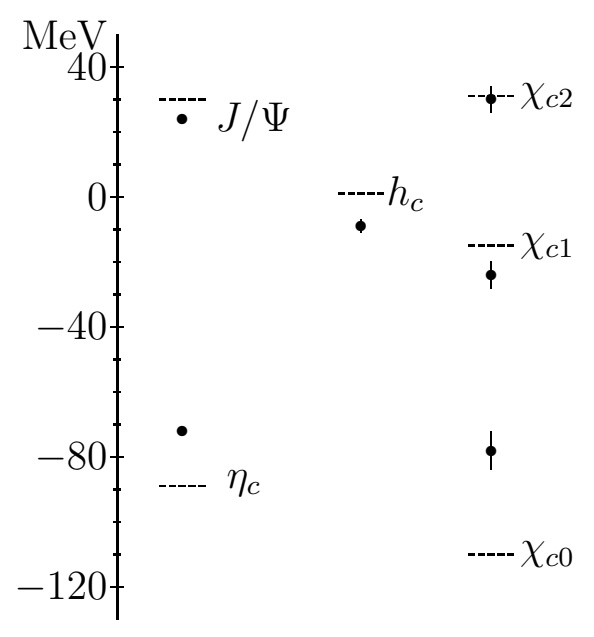

Figure 2: Simulation results for the spin structure of the $J / \Psi$ family, using an inverse lattice spacing of $1.23 \mathrm{GeV}$. The energies of the spin-averaged $\mathrm{S}$ and $\mathrm{P}$ states have been set to zero. Error bars for points are statistical.

$$
\begin{aligned}
& -c_{3} \frac{g}{8\left(M_{c}^{0}\right)^{2}} \boldsymbol{\sigma} \cdot(\boldsymbol{\Delta} \times \mathbf{E}-\mathbf{E} \times \boldsymbol{\Delta})-c_{4} \frac{g}{2 M_{c}^{0}} \boldsymbol{\sigma} \cdot \mathbf{B} \\
& +c_{5} \frac{a^{2} \Delta^{(4)}}{24 M_{c}^{0}}-c_{6} \frac{a\left(\Delta^{(2)}\right)^{2}}{16 n\left(M_{c}^{0}\right)^{2}} .
\end{aligned}
$$

The first two terms in $\delta H$ are spin-independent relativistic corrections and the next two are spin-dependent correction terms which contribute to the $\mathrm{P}$ and $\mathrm{S}$ spin splittings respectively. The last two terms come from finite lattice spacing corrections to the lattice Laplacian and the lattice time derivative. $\boldsymbol{\Delta}$ is the symmetric lattice derivative, $\Delta^{(2)}$ is the lattice form of the Laplacian and $\Delta^{(4)}$ is a lattice version of the continuum operator $\sum D_{i}^{4}$. We used the standard traceless cloverleaf operators for the chromo-electric and magnetic fields, $\mathbf{E}$ and $\mathbf{B}$. The parameter $n$ is introduced to remove instabilities in the heavy quark propagator caused by the highest momentum modes of the theory [1]. For our simulations at $\beta=5.7$ and with a bare mass for the $c$ quark in lattice units of 0.8 , we set $n=4$.

The coupling constants $c_{i}$ appearing in equation (雨) can be calculated by matching NRQCD to full QCD [2, 10]. At tree level all the coefficients are one. The largest radiative corrections are believed to be tadpole contributions [11]. We take care of these by using the method suggested in [11] where all the U's are redefined by

$$
U_{\mu}(x) \rightarrow \frac{U_{\mu}(x)}{u_{0}}
$$

with $u_{0}$ the fourth root of the plaquette (at $\beta=5.7$ we use $u_{0}=0.861$ ). Since the 
cloverleaf expression involves the evaluation of a plaquette this renormalization will have the effect of redefining $\mathbf{E}$ and $\mathbf{B}$ via

$$
\mathbf{E} \rightarrow \frac{\mathbf{E}}{u_{0}^{4}} \quad \mathbf{B} \rightarrow \frac{\mathbf{B}}{u_{0}^{4}}
$$

which will strongly affect spin-dependent splittings. With the dominant tadpole contributions thus removed, we use the tree level values for the $c_{i}$ 's. The only remaining free parameters are the bare quark mass $M_{c}^{0}$ and the bare coupling constant $g$ which appear in the original QCD Lagrangian. All the details of the quark evolution up to this point are identical to those in [3]. In the following some of the technical details differ slightly.

Given the quark propagators in equation (21) it is relatively straightforward to combine them appropriately to form meson propagators with specific quantum numbers. Using the notation of [3] we take $\psi^{\dagger}$ to create a heavy quark and $\chi^{\dagger}$ to create a heavy anti-quark. Then the following interpolating operator creates a meson centred on the point $\vec{x}_{1}$ :

$$
\sum_{\vec{x}_{2}} \psi^{\dagger}\left(\vec{x}_{1}\right) \Gamma\left(\vec{x}_{1}-\vec{x}_{2}\right) \chi^{\dagger}\left(\vec{x}_{2}\right)
$$

Local meson operators are tabulated in [3]. Here we generalise the operators to include 'smearing functions'. For $\mathrm{S}$ states the meson operator $\Gamma$ becomes $\Omega \phi\left(\overrightarrow{x_{1}}-\overrightarrow{x_{2}}\right)$ where $\Omega$ is a $2 \times 2$ matrix in spin space giving the quantum numbers of the meson and $\phi$ is a simple approximation to the wavefunction. For P states, $\phi$ also becomes a $p$ wavefunction, which can be thought of as the derivative of a spherically symmetric function [3]. In general $\Gamma$ is a sum of spin matrices multiplying different smearing functions, generalising the operators in [3]. For the wavefunctions $\phi$ we use here wavefunctions from a $1 / r$ potential with their spread adjusted to match the size of the appropriate meson.

For meson propagators at zero momentum we then have

$$
G_{\text {meson }}(\vec{p}=0, t)=\sum_{\vec{y}_{1}, \vec{y}_{2}} \operatorname{Tr}\left[G_{t}^{\dagger}\left(\vec{y}_{2}\right) \Gamma^{(s k)^{\dagger}}\left(\vec{y}_{1}-\vec{y}_{2}\right) \tilde{G}_{t}\left(\vec{y}_{1}\right)\right]
$$

with

$$
\tilde{G}_{t}(\vec{y}) \equiv \sum_{\vec{x}} G_{t}(\vec{y}-\vec{x}) \Gamma^{(s c)}(\vec{x})
$$

$\Gamma^{s c}(x)$ and $\Gamma^{s k}(x)$ refers to the meson operator $\Gamma(x)=\Omega \phi(x)$ with the smearing function $\phi(x)$ at the source or sink respectively and enumerated by the integer $n_{s c}$ or $n_{s k} . n=1$ corresponds to the ground state meson, $n=2$ to the first radially excited state. $\tilde{G}_{t}$ is obtained using equations (1) and (2) with $\delta_{\vec{x}, 0} \rightarrow \Gamma^{(s c)}(\vec{x})$. The trace is over color and spin. The convolutions are evaluated using Fast Fourier Transforms. 
We also study finite momentum propagators for the ${ }^{1} S_{0}$ meson, given by:

$$
G_{m e s o n}(\vec{p}, t)=\sum_{\vec{y} 1} \operatorname{Tr}\left[G_{t}^{\dagger}\left(\vec{y}_{1}\right) \Omega \tilde{G}_{t}\left(\vec{y}_{1}\right)\right] e^{-i \vec{p} \cdot \vec{y}_{1}}
$$

Using the notation ${ }^{2 S+1} L_{J}$, we have looked at meson propagators for the following states: ${ }^{1} S_{0},{ }^{3} S_{1},{ }^{1} P_{1},{ }^{3} P_{0},{ }^{3} P_{1},{ }^{3} P_{2}$ for both the $\mathrm{E}$ and $\mathrm{T}$ representation and the ${ }^{1} D_{2}$ in the $\mathrm{T}$ representation. For the $\mathrm{S}$ states, smearing functions both for the ground and first radially excited state were used as well as a local $\delta$ function $(n=l o c)$. From this all possible combinations of smearing at the source and sink were formed making a $3 \times 3$ matrix of correlation functions. For the $\mathrm{P}$ and $\mathrm{D}$ states only the ground state smearing function was used at the source. We calculated the dispersion relation for the ${ }^{1} S_{0}$ by looking at the meson propagator for small momentum components using $\left(n_{s c}, n_{s k}\right)=(l o c, l o c)$ and $(1, l o c)$. To maximize our statistics we use all color and spin indices at the source when calculating our meson propagators. For the ${ }^{3} S_{1},{ }^{1} P_{1},{ }^{3} P_{1},{ }^{3} P_{2}$ and ${ }^{1} D_{2}$ we average over polarization directions making a total of $30 \mathrm{~S}, \mathrm{P}$ and $\mathrm{D}$ meson propagators to analyze.

\section{Simulation results}

In the simulation we used 273 quenched gluon field configurations on a $12^{3} \times 24$ lattice at $\beta=5.7$ generously supplied by the UKQCD collaboration[7]. They were fixed to Coulomb gauge using a Fourier accelerated steepest descents algorithm [12] with a cutoff on $[\partial \cdot A]^{2}$ of $10^{-6}$. Due to the relatively small size of the $J / \Psi$ it is possible to use more than one starting site on a spatial slice. We also use more than one starting point in time to increase statistics. In this case we used 8 different spatial origins and 2 different starting times at timeslice 1 and 12. If we bin the spatial origins together we find significant correlation, whereas binning together two propagators with an initial timeslice of 1 and 12 but with the same spatial origin gives little or no correlation at all. For most of our fits we bin together all the correlation functions from a given configuration, except when doing multiple-exponential multiple-correlation fits for the ${ }^{1} S_{0}$ and ${ }^{3} S_{1}$ case. Here we only bin on spatial origin and having the increased sample size from the time direction significantly improves the fit. We also checked, however, that fitting with all data unbinned produces a worse $\chi^{2}$ than when all data is binned, another indicator of spatial correlations.

In NRQCD, as in QCD, there are two free parameters, the bare coupling constant $g$ and the bare quark mass $M_{c}^{0}$. We fix $g$ implicitly when we set the scale $a^{-1}$. To fix $M_{c}^{0}$ we tune so that the simulation result for the kinetic mass of the ${ }^{1} S_{0}$ agrees with the experimental value of the mass of the $\eta_{c}(2.98 \mathrm{GeV})$. 
For this we find $E_{\mathbf{P}}$ for several different momenta of the ${ }^{1} S_{0}$ and fit to the form

$$
E_{\mathbf{P}}-E_{0}=\frac{\mathbf{P}^{2}}{2 M_{k i n}}-C_{1} \frac{\left(\mathbf{P}^{2}\right)^{2}}{8 M_{k i n}^{3}}-\frac{C_{2}}{8 M_{k i n}^{3}} \sum_{i} \mathbf{P}_{i}^{4}
$$

simultaneously for momenta components $(1,0,0),(1,1,0),(1,1,1),(2,0,0)$ in units of $2 \pi / 12 a . M_{k i n}$ is taken to be the rest mass of the $\eta_{c}$. $C_{1}$ should take the value 1.0 in a fully Lorentz invariant theory. Instead we find the value 1.7(1) - this is because of relativistic corrections that have not been included. The mass in the $\mathbf{P}^{4}$ term differs from that in the $\mathbf{P}^{2}$ term by the cube root of 1.7 i.e. $20 \%$. Since we have included no relativistic corrections to the $\mathbf{P}^{4}$ term we would expect it to be correct only to leading order i.e. $30 \%$, and the difference we observe is consistent with that. We expect results closer to 1.0 for the $\Upsilon$ because this is a more non-relativistic system. Indeed there [3] we find a tendency for $C_{1}$ to be larger than 1.0 but consistent with 1.0 within rather larger errors of size 0.3 . The last term in equation (11) is a non-rotationally-invariant term allowed on the lattice but $C_{2}$ is found to be $-0.1(1)$ consistent with zero. This indicates that no discretisation errors are visible in the dispersion relation once the $\mathcal{O}\left(a^{2}\right)$ terms in the heavy quark action have been taken care of in equation (4). A fit with the extra $\mathbf{P}^{6}$ relativistic correction in was tried and no significant signal for it was found. Conversely a fit with just the leading order $\mathbf{P}^{2}$ term in was tried but gave a very poor $Q$ value. This suggests that with the particular momentum components used a fit including terms up to $\mathbf{P}^{4}$ is appropriate. Using a bare quark mass of $a M_{c}^{0}=0.8$ gives $M_{k i n} a=2.429(7)$ or $M_{k i n}=3.0(1) \mathrm{GeV}$ using $a^{-1}=1.23(4) \mathrm{GeV}$. All simulation results quoted here are from using this value of the bare quark mass. The error on the bare quark mass is then of order $10 \%$ from both statistical errors in $a^{-1}$ and systematic errors from higher order relativistic corrections.

In Figures (3) and (4) we show effective masses for the ${ }^{1} S_{0}$ and ${ }^{1} P_{1}$ states respectively. We use the naïve definition $m_{e f f}(t)=-\log (G(t+1) / G(t))$ together with bootstrap errors. From the $\mathrm{S}$ state plots it is clear that smearing has the effect of producing an earlier plateau in the effective mass. Although the statistical errors have increased for the smeared cases as compared to the local-local case the earlier plateau allows fitting to take place closer to the origin and ultimately produces better errors. For the first excited state a plateau cannot be seen for the effective mass and the signal ultimately decays to the ground state. A better transient plateau was seen for the excited $S$ state in the $\Upsilon$ spectrum at $\beta=6.0$ [3]. This reflects the fact that at higher $\beta$ values the excited states have smaller masses in lattice units and last for longer times. For the $\mathrm{P}$ state the signal/noise ratio is much poorer than that for the $\mathrm{S}$ state, as expected. 

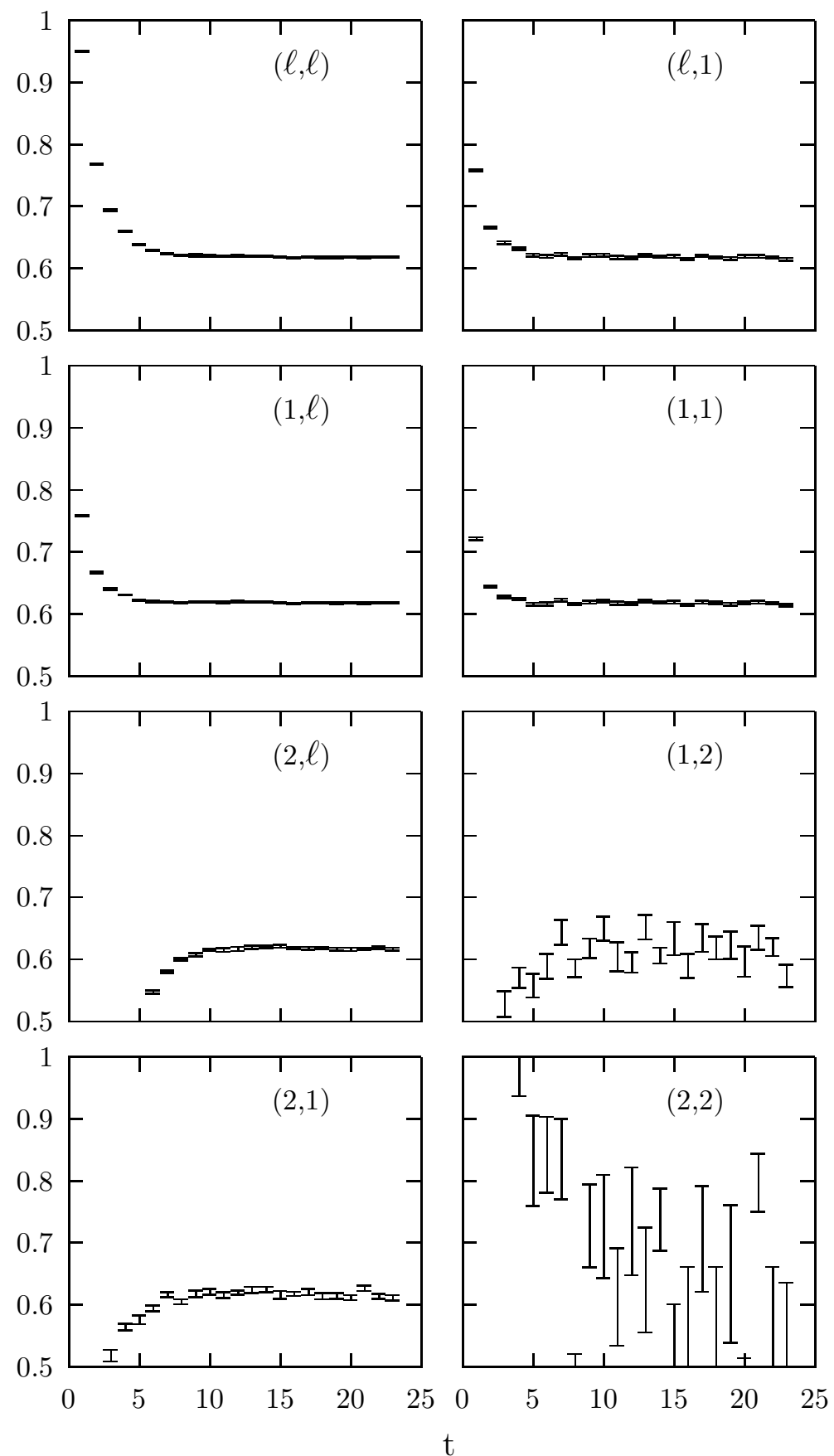

Figure 3: ${ }^{1} S_{0}$ Effective masses by (source, sink). 


\subsection{Fitting Results for the ${ }^{1} S_{0}$ and ${ }^{3} S_{1}$ and the singlet $P$ and $D$ states}

We use a variety of fitting routines to extract high precision ground state masses for the ${ }^{1} S_{0}$ and ${ }^{3} S_{1}$ as well as masses for their first radially excited states. We have used in general the same fitting procedures which are described in more detail in [3].

Multi-exponential fits allow a fit to the correlation function at much earlier times than single-exponential fits, thus reducing the noise. A fit to $n$ exponentials allows confidence in the masses of the first $n-1$ states. Since, as described above, excited states die very rapidly at low $\beta$, it is much harder to get a value for an excited state mass at $\beta=5.7$ than at $\beta=6.0$. This is reflected in our errors. It is also true, however, that the ground state plateau appears earlier and the use of many exponentials to get to early times is not as important at $\beta=5.7$ as at $\beta=$ 6.0 .

The first type of fit we do is that to a matrix of correlation functions:

$$
G_{m e s o n}\left(n_{s c}, n_{s k} ; t\right)=\sum_{k=1}^{N_{e x p}} a\left(n_{s c}, k\right) a^{*}\left(n_{s k}, k\right) e^{-E_{k} \cdot t}
$$

For the $\mathrm{S}$ states we use the combination $n_{s c}=1,2$ and $n_{s k}=1,2$ forming a 2 x 2 matrix. Then we perform fits for $N_{\text {exp }}=2$ and 3 . Our fitting procedure inverts the covariance matrix using the svd algorithm. We have sufficiently good statistics that we are able to keep all eigenvectors of the covariance matrix and achieve a good fit 13.

For the second fit a row of correlation functions is formed and fitted to

$$
G_{\text {meson }}\left(n_{s c}, l o c ; t\right)=\sum_{k=1}^{N_{\text {exp }}} b\left(n_{s c}, k\right) e^{-E_{k} \cdot t}
$$

We use the correlation functions $\left(n_{s c}, n_{s k}\right)$ with $n_{s c}=1,2 n_{s k}=l o c$. Again fits use $N_{\text {exp }}=2,3$.

In Tables 1 and 3 are results from the row and matrix fits for the ${ }^{1} S_{0}$ and ${ }^{3} S_{1}$. The errors stated are those causing a change $\delta \chi^{2}=1$ and we also quote the quality of the fit, Q. For an acceptable fit $\mathrm{Q}$ should be in the range 0.01 to 0.9 and ideally $Q>0.1$. To improve our statistics we only bin correlation functions which start from different spatial origins but not ones which have different starting timeslices. This has little effect on the central value but does increase the $\mathrm{Q}$ value giving us more confidence in the fit.

From both tables it is clear that an accurate ground state mass can be obtained at very early times. Only a $t_{\text {min }}$ of 2 gives an unacceptable $\mathrm{Q}$ for the 2 exponential fit. Adding a 3rd exponential produces an acceptable fit, although we don't take this value because $Q$ increases further as $t_{\text {min }}$ is increased. This contrasts with the higher $t_{\min }$ needed for $\Upsilon$ spectroscopy at $\beta=6.0[3]$. The masses we obtain 


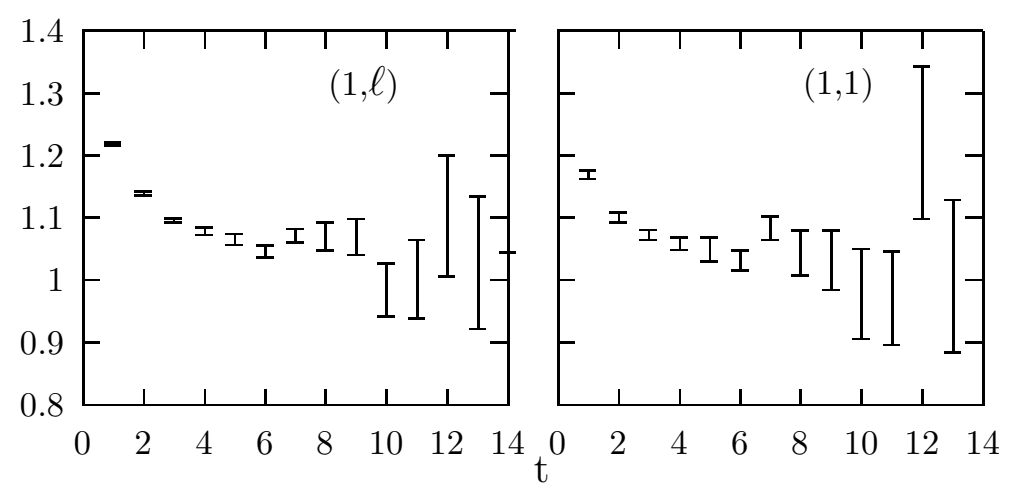

Figure $4:{ }^{1} P_{1}$ Effective masses by (source, sink).

are independent of the type of fitting routine within errors, although the values for $\mathrm{Q}$ are lower for the matrix fits. At this point it is constructive to test how effective the multiple exponential fits are for the ground states at $\beta=5.7$. In Table 2 are values for a single exponential fit to the $\left(n_{s c}, n_{s k}\right)=(1, l o c)$ and $(1,1)$ for the ${ }^{1} S_{0}$ state. In both cases an acceptable $Q$ requires $t_{\text {min }}$ of 6 , significantly larger than for the multiple exponential fit. We choose fitted values $0.6182(7)$ and $0.697(1)$ for the ${ }^{1} S_{0}$ and ${ }^{3} S_{1}$ ground states respectively.

For the first excited state the choice of fitted value is far more difficult. To have confidence in the value we should use a 3 exponential fit although this gives larger errors in the fitted masses. We look for both a steady value in the fitted mass as $t_{\text {min }}$ is changed and a steady value for Q. It is also useful to look at the amplitude for the second excited state in the 3 exponential fit to see at what $t_{\min }$ values it has decayed away.

For the ${ }^{1} S_{0}$ row fit we choose a value 1.17(5) for the excited state mass (average of $\left.t_{\min }=3,4,6\right)$ and from the matrix fit 1.18(4) (average of $t_{\min }=3,4,5$ ). There is then agreement within errors between the two fits and we choose 1.17(5) as the global average. For the ${ }^{3} S_{1}$ state there is a significant deterioration in the $\mathrm{Q}$ values over those for the ${ }^{1} S_{0}$ and the fitting errors are slightly larger. This is presumably a reflection of the additional noise in the ${ }^{3} S_{1}$ channel coming from the ${ }^{1} S_{0}$. For the row fit a value of 1.19(7) (average for $\left.t_{\min }=4,5,6\right)$ is chosen and a value of 1.22(3) (average for $t_{\text {min }}=3,4,5$ ) from the matrix fit. A global average for the excited ${ }^{3} S_{1}$ is chosen to be 1.20(7). All the fitted values are collected in Table 10.

In Tables 1 and 5 are the amplitudes from the various fits for particular values of $t_{\min } / t_{\max }$. The value of $t_{\min } / t_{\max }$ used was that where the fit for the first excited state was closest to the average result quoted above. In both row and matrix fits it was found that the amplitude for a second excited state $(k=$ 3 ) is essentially zero. This indicates that contamination from higher states in our fits is negligible. From the amplitude results we can see that $n_{s c}=1$ has strongest overlap with the ground state and $n_{s c}=2$ has strongest overlap with the first excited state, as planned. Thus our smearing functions are projecting out the 


\begin{tabular}{l|cclllc} 
& $N_{\exp }$ & $t_{\min } / t_{\max }$ & $a E_{1}$ & $a E_{2}$ & $a E_{3}$ & $Q$ \\
\hline fits to (1,loc) & 2 & $2 / 24$ & $0.6171(6)$ & $1.172(6)$ & & $2 \times 10^{-3}$ \\
and (2,loc) & & $3 / 24$ & $0.6178(6)$ & $1.16(1)$ & & 0.65 \\
& & $4 / 24$ & $0.6176(6)$ & $1.16(1)$ & & 0.64 \\
& & $5 / 24$ & $0.6179(7)$ & $1.14(1)$ & & 0.79 \\
& & $6 / 24$ & $0.6182(7)$ & $1.21(5)$ & & 0.94 \\
& \multirow{3}{*}{3} & $2 / 24$ & $0.6183(7)$ & $1.27(8)$ & & 0.93 \\
& & $3 / 24$ & $0.6180(7)$ & $1.15(2)$ & $1.8(6)$ & 0.38 \\
& & $4 / 24$ & $0.6181(6)$ & $1.16(2)$ & $1.8(1.2)$ & 0.79 \\
& & $5 / 24$ & $0.6183(7)$ & $1.30(16)$ & $1.7(6)$ & 0.94 \\
& & $6 / 24$ & $0.6183(7)$ & $1.19(8)$ & $1.8(5)$ & 0.87 \\
$(1,1),(1,2)$ & & $7 / 24$ & $0.6183(7)$ & $1.25(24)$ & $1.8(8)$ & 0.85 \\
\hline fits to & & $3 / 24$ & $0.6185(6)$ & $1.18(2)$ & & 0.06 \\
& & $4 / 24$ & $0.6183(6)$ & $1.17(3)$ & & 0.15 \\
& & $5 / 24$ & $0.6178(6)$ & $1.16(4)$ & & 0.25 \\
& & $6 / 24$ & $0.6177(6)$ & $1.08(6)$ & & 0.16 \\
& & $7 / 24$ & $0.6181(6)$ & $0.90(6)$ & & 0.42 \\
& 3 & $3 / 24$ & $0.6180(6)$ & $1.19(2)$ & $1.6(5)$ & 0.27 \\
& & $4 / 24$ & $0.6178(6)$ & $1.14(4)$ & $2.1(6)$ & 0.23 \\
& & $5 / 24$ & $0.6179(6)$ & $1.21(7)$ & $1.7(6)$ & 0.16 \\
& & $6 / 24$ & $0.6180(6)$ & $1.26(11)$ & $2(1)$ & 0.18 \\
& & $7 / 24$ & $0.6181(6)$ & $0.91(6)$ & $2(1)$ & 0.33 \\
\hline
\end{tabular}

Table 1: Examples of simultaneous multi-exponential fits to the ${ }^{1} S_{0}$ using row and matrix fits respectively.

required state and suppressing the others, although our smearing functions are clearly not optimal. It may be better to use the output wavefunctions to produce input smearing functions in an improved calculation. To illustrate the quality of the multi-exponential fits into early times we have plotted in Figure 5 effective amplitude plots with the fitted parameters quoted in Tables 1 and 5 .

For the $\mathrm{P}$ and $\mathrm{D}$ states multiple exponential fits are not possible because we have included only the ground state smearing function in the simulation. Instead a single exponential fit was performed to the $\left(n_{s c}, n_{s k}\right)=(1,1)$ meson propagators of the ${ }^{1} P_{1}$ and ${ }^{1} D_{2}$. The results are shown in Tables 6 and 0 . Reasonable errors are obtained at $t_{\min }$ values of 6 where single exponential fits were acceptable for the $S$ states. Ratio fits were also done to the ${ }^{1} S_{0}$ in both cases but the results and errors remained the same showing there is no correlation between these states and the ${ }^{1} S_{0}$. To isolate the ground state early on and achieve better errors higher radial smearing functions need to be added. Work has begun on this for the ${ }^{1} P_{1}$ 


\begin{tabular}{c|cccc} 
& $N_{\text {exp }}$ & $t_{\min } / t_{\max }$ & $a E_{1}$ & $Q$ \\
\hline fits to $(1$, loc $)$ & 1 & $5 / 24$ & $0.6188(8)$ & 0.01 \\
& & $6 / 24$ & $0.6184(8)$ & 0.66 \\
& & $7 / 24$ & $0.6183(8)$ & 0.72 \\
\hline fits to $(1,1)$ & 1 & $4 / 24$ & $0.6184(8)$ & 0.05 \\
& & $5 / 24$ & $0.6181(8)$ & 0.22 \\
& & $6 / 24$ & $0.6181(8)$ & 0.18 \\
& & $7 / 24$ & $0.6182(8)$ & 0.15 \\
\hline
\end{tabular}

Table 2: Examples of single exponential fits to the ${ }^{1} S_{0}$.

\begin{tabular}{|c|c|c|c|c|c|c|}
\hline & $N_{e x p}$ & $t_{\min } / t_{\max }$ & $a E_{1}$ & $a E_{2}$ & $a E_{3}$ & $Q$ \\
\hline \multirow{12}{*}{$\begin{array}{l}\text { fits to }(1, \text { loc }) \\
\text { and }(2, \text { loc })\end{array}$} & 2 & $2 / 24$ & $0.6951(8)$ & $1.247(7)$ & & $4 \times 10^{-5}$ \\
\hline & & $3 / 24$ & $0.6961(8)$ & $1.23(1)$ & & 0.23 \\
\hline & & $4 / 24$ & $0.6958(9)$ & $1.22(2)$ & & 0.23 \\
\hline & & $5 / 24$ & $0.6961(9)$ & $1.18(2)$ & & 0.46 \\
\hline & & $6 / 24$ & $0.6966(9)$ & $1.21(5)$ & & 0.56 \\
\hline & & $7 / 24$ & $0.6968(10)$ & $1.25(8)$ & & 0.56 \\
\hline & 3 & $2 / 24$ & $0.6964(9)$ & $1.21(4)$ & $1.9(9)$ & 0.10 \\
\hline & & $3 / 24$ & $0.6957(9)$ & $1.20(4)$ & $1.9(1.4)$ & 0.17 \\
\hline & & $4 / 24$ & $0.6964(10)$ & $1.16(5)$ & $1.9(1.3)$ & 0.47 \\
\hline & & $5 / 24$ & $0.6967(10)$ & $1.22(8)$ & $1.9(5)$ & 0.55 \\
\hline & & $6 / 24$ & $0.6966(7)$ & $1.19(6)$ & $1.9(3)$ & 0.41 \\
\hline & & $7 / 24$ & $0.6969(10)$ & $1.25(16)$ & $1.9(2)$ & 0.40 \\
\hline \multirow{10}{*}{$\begin{array}{l}\text { fits to } \\
(1,1),(1,2) \\
(2,1),(2,2)\end{array}$} & 2 & $3 / 24$ & $0.6970(8)$ & $1.22(1)$ & & 0.04 \\
\hline & & $4 / 24$ & $0.6967(8)$ & $1.21(3))$ & & 0.05 \\
\hline & & $5 / 24$ & $0.6965(8)$ & $1.24(5)$ & & 0.07 \\
\hline & & $6 / 24$ & $0.6966(8)$ & $1.31(9)$ & & 0.09 \\
\hline & & $7 / 24$ & $0.6967(9)$ & $0.95(8)$ & & 0.08 \\
\hline & 3 & $3 / 24$ & $0.6966(8)$ & $1.23(2)$ & $1.7(6)$ & 0.08 \\
\hline & & $4 / 24$ & $0.6965(8)$ & $1.20(3)$ & $1.8(6)$ & 0.06 \\
\hline & & $5 / 24$ & $0.6964(8)$ & $1.23(4)$ & $2.0(2.8)$ & 0.04 \\
\hline & & $6 / 24$ & $0.6969(8)$ & $1.46(13)$ & $1.8(1.3)$ & 0.06 \\
\hline & & $7 / 24$ & $0.6967(9)$ & $1.00(9)$ & $1.9(1.3)$ & 0.07 \\
\hline
\end{tabular}

Table 3: Examples of simultaneous multi-exponential fits to the ${ }^{3} S_{1}$ using row and matrix fits respectively. 


\begin{tabular}{l|ccll}
\multicolumn{1}{r|}{ Fit } & $t_{\min } / t_{\max }$ & $\mathrm{k}$ & $a\left(n_{s c, s k}=1, k\right)$ & $a\left(n_{s c, s k}=2, k\right)$ \\
\hline$N_{\text {exp }}=2$ & $4 / 24$ & 1 & $0.681(1)$ & $-0.1188(8)$ \\
for ${ }^{1} S_{0}$ & & 2 & $0.18(9)$ & $0.52(2)$ \\
\hline$N_{\exp }=2$ & $5 / 24$ & 1 & $0.700(3)$ & $-0.164(1)$ \\
for ${ }^{3} S_{1}$ & & 2 & $0.29(2)$ & $0.53(5)$ \\
\hline
\end{tabular}

Table 4: Examples of fit results for amplitudes $a\left(n_{s c, s k}, k\right)$

\begin{tabular}{l|cclc}
\multicolumn{1}{r|}{ Fit } & $t_{\text {min }} / t_{\text {max }}$ & $\mathrm{k}$ & $b\left(n_{s c}=1, k\right)$ & $b\left(n_{s c}=2, k\right)$ \\
\hline$N_{\text {exp }}=2$ & $4 / 24$ & 1 & $0.1037(7)$ & $-0.0184(4)$ \\
for ${ }^{1} S_{0}$ & & 2 & $0.032(3)$ & $0.064(2)$ \\
\hline$N_{\text {exp }}=2$ & $5 / 24$ & 1 & $0.103(1)$ & $-0.0253(4)$ \\
for ${ }^{3} S_{1}$ & & 2 & $0.036(7)$ & $0.069(3)$ \\
\hline
\end{tabular}

Table 5: Examples of fit results for amplitudes $b\left(n_{s c}, k\right)$

state.

\subsection{Fits to Spin Splittings}

As described earlier, spin splittings are very dependent on the tadpole improved coupling constants $c_{i}$. This makes the spin-splittings a good test of the tadpoleimprovement scheme. It is also true that potential models find it hard to produce spin-splittings in agreement with experiment so we would hope that they are also a good test of the differences between a full calculation in QCD, such as ours, and a potential model.

Since meson correlation functions of given $l$ from the same configuration are highly correlated we produce a bootstrap ensemble of ratios of correlation func-

\begin{tabular}{l|cccc} 
& $N_{\exp }$ & $t_{\min } / t_{\max }$ & $a E_{1}$ & $Q$ \\
\hline fits to $(1,1)$ & 1 & $3 / 24$ & $1.059(4)$ & 0.45 \\
& & $4 / 24$ & $1.052(5)$ & 0.68 \\
& & $5 / 24$ & $1.049(7)$ & 0.66 \\
& & $6 / 24$ & $1.046(9)$ & 0.62 \\
& & $7 / 24$ & $1.048(14)$ & 0.55 \\
\hline
\end{tabular}

Table 6: Example of a ${ }^{1} P_{1}$ fit. 


\begin{tabular}{c|cccc} 
& $N_{\text {exp }}$ & $t_{\min } / t_{\max }$ & $a E_{1}$ & $Q$ \\
\hline fits to $(1,1)$ & 1 & $3 / 24$ & $1.35(1)$ & 0.62 \\
& & $4 / 24$ & $1.32(2)$ & 0.77 \\
& & $5 / 24$ & $1.30(3)$ & 0.78 \\
& & $6 / 24$ & $1.26(5)$ & 0.78 \\
& & $7 / 24$ & $1.26(9)$ & 0.72 \\
\hline
\end{tabular}

Table 7: Example of a ${ }^{1} D_{2}$ fit.
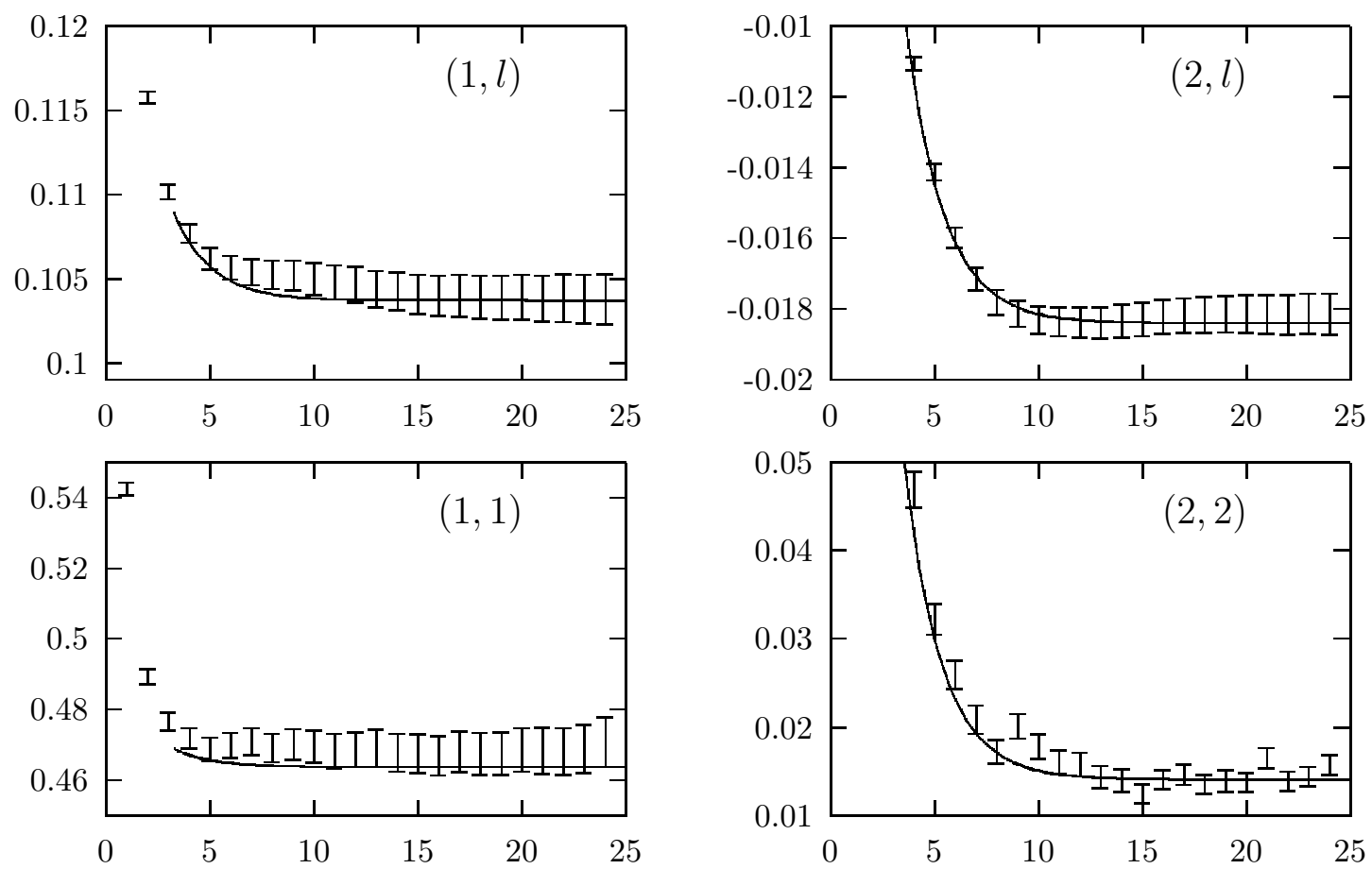

Figure 5: ${ }^{1} S_{0}$ Effective amplitudes $G(t) \cdot e^{E_{1} \cdot t}$ from two-exponential row fits $(1, \ell)$ $(2, \ell)$ and two-exponential matrix fits $(1,1)(2,2)$ with $t_{\min }=4, t_{\max }=24$. 


\begin{tabular}{l|cccc} 
Splitting & $N_{\text {exp }}$ & $t_{\min } / t_{\max }$ & $a \delta E$ & $Q$ \\
\hline${ }^{3} S_{1}-{ }^{1} S_{0}$ & 1 & $4 / 24$ & $0.0794(3)$ & $4.0 \times 10^{-5}$ \\
& & $6 / 24$ & $0.0784(4)$ & 0.35 \\
& & $8 / 24$ & $0.0784(4)$ & 0.32 \\
& & $10 / 24$ & $0.0783(5)$ & 0.21 \\
& & $12 / 24$ & $0.0778(6)$ & 0.25 \\
\hline${ }^{3} P_{2 E}-{ }^{3} P_{0}$ & 1 & $3 / 13$ & $0.090(2)$ & 0.94 \\
& & $4 / 13$ & $0.089(4)$ & 0.91 \\
& & $5 / 13$ & $0.090(6)$ & 0.85 \\
& & $6 / 13$ & $0.086(9)$ & 0.81 \\
\hline${ }^{3} P_{2 E}-{ }^{3} P_{1}$ & 1 & $3 / 13$ & $0.045(1)$ & 0.99 \\
& & $4 / 13$ & $0.046(3)$ & 0.99 \\
& & $5 / 13$ & $0.045(4)$ & 0.99 \\
& & $6 / 13$ & $0.044(6)$ & 0.97
\end{tabular}

Table 8: Examples of ratio fits for spin-splittings

tions to find spin splittings. From this we fit to a single exponential

$$
\operatorname{Ratio}(t)=A e^{-\delta E t}
$$

We use correlation functions with $\left(n_{s c}, n_{s k}\right)=(1,1)$ and bin on time and spatial origin. We find very high $\mathrm{Q}$ values in general. Shown in Table 8 are values obtained for various combinations of spin-splittings using equation (14). The $\delta E$ obtained for the ${ }^{3} S_{1}$ to ${ }^{1} S_{0}$ ratio fit is in agreement with that obtained from the separate row and matrix fits of Tables 1 and 3. To estimate $\delta E$ for higher radial excitations we have used a correlated $\delta E$ fit. This is a fit to the form

$$
\begin{aligned}
& G_{\text {meson } A}\left(n_{s c}, l o c ; t\right)=\sum_{k=1}^{N_{\text {exp }}} c_{A}\left(n_{s c}, k\right) e^{-E_{k}^{A} \cdot t} \\
& G_{\text {meson } B}\left(n_{s c}, l o c ; t\right)=c_{B}\left(n_{s c}, 1\right) e^{-\left(E_{1}^{A}+\delta E\right) \cdot t}+\sum_{k=2}^{N_{\text {exp }}} c_{B}\left(n_{s c}, k\right) e^{-E_{k}^{B} \cdot t}
\end{aligned}
$$

with $n_{s c}=1,2$ for each meson. The results shown in Table 9 show that the ${ }^{3} S_{1}-{ }^{1} S_{0}$ splitting can be obtained at early times with smaller errors than in the ratio fit. Presumably extra excited states have been absorbed in the extra terms in the correlated fit. We are unable to obtain a clear signal for a $2 \mathrm{~S}$ hyperfine splitting although the correlated $\delta E$ fit above and the individual matrix fits give an indication of such a splitting at early times. 


\begin{tabular}{cccccc}
$t_{\min } / t_{\max }$ & $1^{1} S_{0}$ & $2^{1} S_{0}$ & $1^{3} S_{1}-1^{1} S_{0}$ & $2^{3} S_{1}$ & $Q$ \\
\hline $3 / 24$ & $0.6179(6)$ & $1.17(1)$ & $0.0779(3)$ & $1.23(1)$ & 0.29 \\
$4 / 24$ & $0.6178(6)$ & $1.17(1)$ & $0.0778(3)$ & $1.24(2)$ & 0.33 \\
$5 / 24$ & $0.6180(6)$ & $1.16(2)$ & $0.0777(3)$ & $1.19(2)$ & 0.72 \\
$6 / 24$ & $0.6183(6)$ & $1.20(4)$ & $0.0780(4)$ & $1.20(4)$ & 0.88 \\
$7 / 24$ & $0.6183(7)$ & $1.20(6)$ & $0.0781(4)$ & $1.20(6)$ & 0.82 \\
$8 / 24$ & $0.6184(7)$ & $1.16(11)$ & $0.0781(4)$ & $1.25(13)$ & 0.76 \\
\hline
\end{tabular}

Table 9: Example of correlated $\delta E$ fit for the ${ }^{3} S_{1}$ and ${ }^{1} S_{0}$ states

\begin{tabular}{c|c} 
& Simulation Results \\
\hline $1^{1} S_{0}$ & $0.6182(7)$ \\
$1^{3} S_{1}$ & $0.697(1)$ \\
$2^{1} S_{0}$ & $1.17(5)$ \\
$2^{3} S_{1}$ & $1.20(7)$ \\
$1^{1} P_{1}$ & $1.05(1)$ \\
$1^{1} D_{2}$ & $1.30(4)$ \\
\hline${ }^{3} S_{1}-{ }^{1} S_{0}$ & $0.0782(4)$ \\
${ }^{3} P_{2}-{ }^{3} P_{0}$ & $0.088(8)$ \\
${ }^{3} P_{2}-{ }^{3} P_{1}$ & $0.044(5)$ \\
${ }^{3} P_{1}-{ }^{3} P_{0}$ & $0.044(3)$ \\
${ }^{3} P_{C M}-{ }^{1} P_{1}$ & $0.010(1)$ \\
\hline
\end{tabular}

Table 10: Fitted dimensionless energies. 


\section{Comparison with Experiment}

In Table 10 we give the dimensionless splittings obtained from our fitting procedure. To compare simulation results to experiment it is necessary to fix the scale $a^{-1}$. We choose the spin-averaged 1P-1S splitting to do this. By spin-averaged splitting we mean the splitting between spin-averaged states. The spin-averaged $\mathrm{S}$ state has mass $0.25 \times\left[3 m\left({ }^{3} S_{1}\right)+m\left({ }^{1} S_{0}\right)\right]$. The spin-averaged $\mathrm{P}$ state has either the mass of the ${ }^{1} P_{1}$ or mass $1 / 9 \times\left[5 m\left({ }^{3} P_{2}\right)+3 m\left({ }^{3} P_{1}\right)+m\left({ }^{3} P_{0}\right)\right]$. These two $\mathrm{P}$ masses are the same in potential models and experimentally they do seem to be very close although the mass of the ${ }^{1} P_{1}$ needs confirmation [14. In our simulation the two masses are slightly different (see Table [10). We will use $m\left({ }^{1} P_{1}\right)$ because of the previously noted disagreement with experiment in the $\mathrm{P}$ fine structure. The difference in value of the $a^{-1}$ s obtained is within the statistical error.

The spin-averaged 1P-1S splitting has the advantage of being independent of any errors in spin-dependent terms and of being experimentally known to be independent of the heavy quark mass in the $c, b$, region. This gives much less systematic uncertainty than, for example, in light hadron spectrum determinations of $a^{-1}$. In the $\Upsilon$ spectrum calculation [3] it was possible to see a difference in $a^{-1}$ between that fixed from the $2 \mathrm{~S}-1 \mathrm{~S}$ splitting and that fixed from the $1 \mathrm{P}-1 \mathrm{~S}$ splitting. Here both our statistical error on the $2 \mathrm{~S}$ state and our expected systematic error from relativistic corrections are too large for this to be possible.

Using the values in Table 10 we find $a^{-1}=1.23(4) \mathrm{GeV}$ from the $1 \mathrm{P}\left({ }^{1} P_{1}\right)-1 \mathrm{~S}$ splitting. In Table 11 we compare the splittings obtained from this simulation with experimental results. The results are plotted in Figures (1) and (2). It is important to remember that there is a potential $30-40 \mathrm{MeV}$ systematic error in all splittings coming from relativistic corrections not included in the heavy quark action. Table 11 and the figures do not include the statistical error in $a^{-1}$ in their quoted errors since all the splittings are correlated. Table 11 does, however, include this error for the hyperfine splitting since this is very sensitive to shifts in the bare quark mass allowed by uncertainties in $a^{-1}$ (the hyperfine splitting behaves as $1 / M_{Q}$ in perturbation theory, see equation (16) below). Using the $\chi_{c}$ average for $1 \mathrm{P}$ would give $a^{-1}=1.20$, at the lower end of the range for $a^{-1}$ from the ${ }^{1} P_{1}$.

As discussed earlier, the statistical error on the $2 \mathrm{~S}$ state is too large to see any significance in the fact that it is slightly higher than experiment. The direction of the slight disagreement is the same as that for the $\Upsilon$ spectrum [3]. There it seems clear that the correction of $\mathcal{O}\left(a^{2}\right)$ errors in the gluon action and unquenching will produce agreement with experiment [6, 16]. To test this for the $\Psi$ we will need to reduce the statistical errors and systematic errors from the heavy quark action in the $2 \mathrm{~S}$ state.

The expected shift in the 1S state from gluonic $\mathcal{O}\left(a^{2}\right)$ effects is 0.006 in lattice units for this simulation. This is calculated either perturbatively from the wavefunction at the origin [6] or non-perturbatively using a lattice potential model 


\begin{tabular}{c|ll} 
& Simulation Results $[\mathrm{GeV}]$ & Experiment $[\mathrm{GeV}]$ \\
\hline $2^{1} S_{0}-1{ }^{1} S_{0}$ & $0.68(6)$ & \\
$2^{3} S_{1}-1{ }^{3} S_{1}$ & $0.62(8)$ & $0.589(1)$ \\
${ }^{1} D_{2}-{ }^{1} S_{0}$ & $0.84(5)$ & \\
${ }^{3} D_{1}-{ }^{1} S_{0}$ & & $0.791(3)$ \\
\hline${ }^{3} S_{1}-{ }^{1} S_{0}$ & $0.096(2)$ & $0.118(2)$ \\
${ }^{3} P_{2}-{ }^{3} P_{0}$ & $0.11(1)$ & $0.141(1)$ \\
${ }^{3} P_{2}-{ }^{3} P_{1}$ & $0.054(6)$ & $0.0456(1)$ \\
${ }^{3} P_{C M}-{ }^{1} P_{1}$ & $0.012(1)$ & $0.0008(3)^{*}$ \\
\hline
\end{tabular}

Table 11: NRQCD spectrum results and comparison with experiment for $a^{-1}=$ $1.23 \mathrm{GeV}$ and $a M_{c}^{0}=0.8 .{ }^{*}$ requires confirmation.

[15]. It is less than the shift for the $\Upsilon$ at the same value of $\beta$ since the $J / \Psi$ is larger. The $1 \mathrm{P}$ state does not shift, since it is not sensitive to perturbations at the origin. The change in the $1 \mathrm{P}-1 \mathrm{~S}$ splitting would then cause the derived $a^{-1}$ to change upwards to $1.25(4) \mathrm{GeV}$ if gluonic $\mathcal{O}\left(a^{2}\right)$ effects were corrected. This is still within $1 \sigma$ of the original value. The expected shift in the $\psi^{\prime}$ state is 0.005 so the change in the $2 \mathrm{~S}-1 \mathrm{~S}$ splitting would be completely negligible compared to its statistical error.

The value for $a^{-1}$ is clearly different from that from $\Upsilon[16]$ or light hadron [8] spectroscopy at the same value of $\beta$. In the quenched approximation we would expect $a_{b \bar{b}}^{-1}>a_{c \bar{c}}^{-1}>a_{m_{\rho}}^{-1}$, reflecting the ordering of the momentum scales appropriate to the different quantities. In current results, the first inequality holds but the second one does not [16]; this may reflect $\mathcal{O}(a)$ errors in present light hadron spectroscopy. Further calculations at different values of $a$ will resolve this problem.

The ${ }^{1} D_{2}$ state whose mass we have calculated is rather higher than that found for the $\psi(3770)$, thought to be a ${ }^{3} D_{1}$ state. From the spin splittings alone you would expect this difference. The $\psi(3770)$ is also above threshold for decay to $\bar{D} D$ so quenching might have a significant effect on masses in this region, although the ratio of the width of the $\psi(3770)$ to its mass is still less than $1 \%$. The ${ }^{3} D_{1}$ has the same $J^{P C}$ quantum numbers as the ${ }^{3} S_{1}$ and will appear as a third excited state in that channel. In order to observe such a state the cross-correlation between the meson correlators ${ }^{3} S_{1}$ and the ${ }^{3} D_{1}$ would have to be calculated and we have not attempted to do this here.

Values for the wave function at the origin can be obtained as discussed in ref. [3]. If we include the $(l o c, l o c)$ correlation function in a multi-exponential row fit we obtain a value of $a^{3 / 2} \psi(0)$ for the $J / \Psi$ of 0.1535 . This method does not yield a stable value for the excited states since the $(l o c, l o c)$ correlation function does not distinguish different states very readily. A better method is take a 
ratio of amplitudes from row and matrix fits [3]. We use $b\left(n_{s c}, m\right) / a\left(n_{s c}, m\right)$ and concentrate on the diagonal entries i.e. $n_{s c}=m=1$ for $J / \Psi$ and $n_{s c}=2$ for $\psi^{\prime}$. This gives $a^{3 / 2} \psi(0)=0.148(2)$ for $J / \Psi$ and $0.13(1)$ for $\psi^{\prime}$.

The leptonic width can be calculated from $\psi(0)$ using the Van-Royen Weisskopf formula 17 at leading order. We obtain $5.4(5) \mathrm{keV}$ for the $J / \Psi$ in good agreement with the experimental value of $5.3(3) \mathrm{keV}$. The error we quote is dominated by the error in $a^{-1}$ since this appears cubed. In principle we expect large corrections $(\approx 30 \%)$ to our value when a current correctly matched to the continuum current is included, instead of the naïve lowest order current that we have used. We should apply small-components corrections to the current [18] as well as a lattice-to-continuum renormalisation. The agreement with experiment should thus not be taken to be very significant at this stage. For the $\psi^{\prime}$ the agreement with experiment is not so good. The experimental value is less than half of that for the $1 \mathrm{~S}$ and yet we obtain a ratio of 0.7 to the $1 \mathrm{~S}$. This trend for excited states to have too large a value for $\psi(0)$ is again similar to that found in the $\Upsilon$ case. On improving the systematic error in our currents we would hope to notice an improvement here unless it is a feature of the quenched approximation.

Spin splittings have been calculated for the ground $\mathrm{S}$ and $\mathrm{P}$ states. These are shown in detail in Figure (2). The agreement with experiment is good within expected systematic errors of $30-40 \mathrm{MeV}$. This would not be possible without tadpole-improvement of the spin-dependent terms. It was clear from the $\Upsilon$ spectrum[3] that splittings without tadpole improvement were about half the size of those with tadpole improvement. This would be an even bigger effect here where $\beta$ and $u_{0}$ are smaller. There is nevertheless some disagreement with experiment in Figure (2), and it is useful to find the source of this. There are sufficient experimental results for charmonium that the system provides a good test of the systematic removal of sources of error.

From Table 11 we can see that the hyperfine splitting $M\left({ }^{3} S_{1}\right)-M\left({ }^{1} S_{0}\right)$ has a very small statistical error. The difference from experiment then shows up clearly and is presumably a result of our systematic errors. There is again a $30-40 \mathrm{MeV}$ systematic error from higher order relativistic, discretisation and radiative corrections to the heavy quark action. This would be quite sufficient to explain the difference. Relativistic corrections are documented in [1]. The radiative corrections are $\mathcal{O}\left(g^{2}\right)$ corrections to the coefficient of the $\sigma \cdot \vec{B}$ term beyond tadpole improvement. The discretisation errors are $\mathcal{O}\left(a^{2}\right)$ errors in the $\vec{B}$ field and the hyperfine splitting is rather sensitive to these, as discussed below. We also expect quenching to have a significant effect, however. A comparison of $\Upsilon$ results on quenched and unquenched configurations shows an increase in the hyperfine splitting when unquenched (to 3 flavours) of between $30 \%$ and $50 \%$ [6, 16]. This can be explained largely on the basis of the difference between quenched and unquenched coupling constants and wavefunctions appearing in 
the perturbative formula for the hyperfine splitting,

$$
\Delta M_{\mathrm{hfs}}=\frac{32 \pi \alpha_{s}\left(M_{Q}\right)}{9 M_{c}^{2}}|\psi(0)|^{2} .
$$

For the $J / \Psi$ case we might expect a similar shift of the hyperfine splitting on going to the full theory and this again would be sufficient to explain fully the deviation from experiment. One problem here is that the perturbative formulae are not quite as reliable as in the $\Upsilon$ case [6].

A calculation of the $\bar{c} c$ hyperfine splitting by the Fermilab group [19 gives a somewhat smaller value than ours. They use an improved Wilson fermion action for the heavy quarks and this approach has different systematic errors than ours.

The case of the $\mathrm{P}$ state fine splittings is much more complicated, with an expected interplay of short and long range effects. In a potential model approach [20] two terms contribute - one proportional to $\langle\vec{L} \cdot \vec{S}\rangle$ and the other proportional to $\left\langle S_{12}\right\rangle$ where $S_{12}=4\left[3\left(\overrightarrow{s_{1}} \cdot \hat{n}\right)\left(\overrightarrow{s_{2}} \cdot \hat{n}-\overrightarrow{s_{1}} \cdot \overrightarrow{s_{2}}\right)\right]$. Here $s_{1}$ and $s_{2}$ are the spins of the heavy quarks and $\hat{n}$ is an arbitrary unit vector. Evaluating these expectation values for ${ }^{3} P_{0},{ }^{3} P_{1}$ and ${ }^{3} P_{2}$ states of equal mass quarks allows us to compare ratios of the splittings, since the the expectation values of potentials that accompany these terms are the same for all $P$ states. A useful ratio [20] is

$$
r=\frac{M\left(\chi_{2}\right)-M\left(\chi_{1}\right)}{M\left(\chi_{1}\right)-M\left(\chi_{0}\right)}
$$

Experimental values are $0.48(1)$ for $c \bar{c}, 0.66(2)$ for $b \bar{b}(1 \mathrm{P})$ and $0.58(3)$ for $b \bar{b}(2 \mathrm{P})$. From a comparison of possible potentials to experiment the conventional picture emerges in which the spin-orbit potential appearing with $\vec{L} \cdot \vec{S}$ has both short and long range pieces, whereas the tensor potential appearing with $S_{12}$ has only a short range piece. The short-range pieces can be related to 1-gluon exchange in perturbation theory and behave like $1 / R^{3}$. The long-range piece comes from the scalar confining potential. Spin-dependent potentials can be extracted on the lattice from expectation values of Wilson loops with $E$ and $B$ field insertions along the time lines on either side. There it becomes clear that the 'same-side' spin-orbit potential is long-range, whereas the 'opposite-side' is short-range, as is the tensor potential 21.

We can extract values for the above ratio $r$ of $\mathrm{P}$ spin splittings from our simulation and we find $1.2(2)$ for $c \bar{c}$, clearly too large. The $b \bar{b}(1 \mathrm{P})$ result at $\beta=$ 6.0 [3] is $0.7(3)$, which is consistent with experiment, but at $\beta=5.7$ we obtain 1.4(4) [9]. It seems likely then that the disagreement with experiment arises from discretisation errors. At low $\beta$ the predominant spin-dependent potential is the long-range spin-orbit piece, the shorter range pieces are not well resolved (compare [22] and [23], for example). A pure $\vec{L} \cdot \vec{S}$ potential would give a value for $r$ of 2 [20] (the pure tensor would give -0.4). In potential model language the long-range $\vec{L} \cdot \vec{S}$ term has undue dominance in our simulation. We also find that 
the overall size of the $\mathrm{P}$ spin splittings, set by $M\left(\chi_{2}\right)-M\left(\chi_{0}\right)$, is too small. For $b \bar{b}$ at $\beta=6.0$ this splitting was on the low side but in agreement with experiment within the error [3]. For $b \bar{b}$ at $\beta=5.7$ we obtain a result which is much too small [9]. Future calculations will concentrate on correcting discretisation errors to see if the results for charmonium at low $\beta$ improve.

Another possible discretisation error shows up in the fact that the centre of mass of the ${ }^{3} P$ states comes out above the ${ }^{1} P_{1}$. This happens both for this calculation and that of the $\Upsilon$ spectrum [3], but in both cases at a level within the expected systematic errors. One might expect, for example, that the hyperfine $\vec{S} \cdot \vec{S}$ interaction would contribute such a term to $P$ states even in the absence of a wavefunction at the origin (see equation (16)) if the $B$ field was smeared out over a plaquette as it is here. Experimental evidence so far indicates that there is no such splitting [14, although it awaits confirmation.

It seems likely that errors from the quenched approximation (and from discretisation) are not so large for the $\mathrm{P}$ fine structure as for the S hyperfine splitting because the latter is determined by very short range phenomena. The hyperfine splitting can be thought of as resulting from delta-function $\overrightarrow{S_{1}} \cdot \overrightarrow{S_{2}}$ potential at the origin (see equation (16)), where S states have significant wave function. The quenched approximation causes larger effects at short distance scales because it appears, perturbatively, as an incorrect running of the coupling constant $\alpha(R)$ down to the origin from some $\bar{R}$ which is the important separation for quark and antiquark in the $1 \mathrm{P}-1 \mathrm{~S}$ splitting which is used to set the scale. $\mathrm{P}$ states have no wavefunction at the origin and in addition the short-range pieces of the relevant spin-dependent potentials have longer range than the delta function hyperfine potential. This should mean that the $\mathrm{P}$ fine structure can be determined accurately in a quenched calculation by a systematic improvement on this calculation, without having to unquench.

\section{Conclusions}

This represents a first calculation of the $c \bar{c}$ spectrum using NRQCD with spindependent terms. We include the leading relativistic and discretisation errors with tadpole-improved coefficients. We find a value of the lattice spacing from the $1 \mathrm{P}-1 \mathrm{~S}$ splitting which is different from that of the $\Upsilon$ on the same configurations 9. This is a clear indication of an effect from the quenched approximation. Another effect seen in the $\Upsilon$ spectrum itself, the difference in $a^{-1}$ from the $2 \mathrm{~S}-1 \mathrm{~S}$ and $1 \mathrm{P}-1 \mathrm{~S}$ splittings, is not visible here above the statistical noise in the $2 \mathrm{~S}$ state.

With tadpole-improvement, the spin splittings agree with experiment at the level of the systematic error that we expect. The trend of these systematic errors is the same as that for the $\Upsilon$ spectrum and we would expect that, on including higher order terms, we could obtain better agreement. It seems likely that the major errors at present are discretisation effects and future calculations 
will correct for these. One very good feature of the $c \bar{c}$ spectrum is that all the radial ground state $\mathrm{S}$ and $\mathrm{P}$ masses are known experimentally and so they can be used to gauge the effect of systematic improvement. Further calculations of the $c \bar{c}$ spectrum on lattices of different lattice spacing and on unquenched configurations would also provide useful checks of the systematic errors. A value for $\alpha_{s}$ could be extracted from the 1P-1S splitting in the same way that it was done using the $\Upsilon$ calculation [6] and a comparison with results from Wilson fermions [24, 25] made.

Calculations of the $B_{c}$ spectrum combining $b$ and $c$ propagators on these configurations will be reported shortly [9].

Acknowledgements This calculation was performed at the Atlas Centre under grant GR/J18927 from the UK PPARC. AJL is also grateful to PPARC for a studentship, CTHD for support under grant GR/J21231 and JSl for a Visiting Fellowship to Glasgow while this work was being completed. This work was supported in part by grants from the U.S.Department of Energy (DE-FC0585ER250000, DE-FG05-92ER40742, DE-FG02-91ER40690), and the National Science Foundation. We thank UKQCD for making their configurations available to us, and in particular David Henty who helped us to read them. Finally, we wish to acknowledge fruitful discussions with Aida El-Khadra, Paul Mackenzie, Colin Morningstar and Beth Thacker.

\section{References}

[1] G. P. Lepage and B. A. Thacker, in Field Theory on the Lattice, Proceedings of the International Symposium, Seillac, France, 1987, edited by A. Billoire et al. [Nucl. Phys. B (Proc. Suppl.) 4 199, (1988)]; B. A. Thacker and G. P. Lepage, Phys. Rev. D 43, 196 (1991).

[2] G. P. Lepage, L. Magnea, C. Nakhleh, U. Magnea and K. Hornbostel, Phys. Rev. D 46, 4052 (1992).

[3] C. T. H. Davies, K. Hornbostel, A. Langnau, G. P. Lepage, A. Lidsey, J. Shigemitsu and J. Sloan, Phys. Rev. D50 (1994) 6963

[4] G. P. Lepage and J. Sloan, poster sessions at LAT93; NRQCD collaboration, Nucl. Phys. B (Proc. Suppl.) 34, 417 (1994).

[5] C.T.H. Davies et al., Phys. Rev. Lett. 73 (1994) 2654.

[6] C. T. H. Davies et al., Phys. Lett. B 345 (1995) 42.

[7] We are very grateful to the UKQCD collaboration for the use of these configurations. They were generated on a Meiko Computing Surface with support from the UK SERC, the University of Edinburgh and Meiko Ltd. 
[8] F. Butler et al, Nucl. Phys. B 430 (1994) 179.

[9] NRQCD collaboration, in preparation.

[10] C. J. Morningstar, Phys. Rev. D 48, 2265 (1993); Nucl. Phys. B (Proc. Suppl.) 34, 425 (1994); Phys. Rev. D 50 (1994) 5902.

[11] G. P. Lepage and P. B. Mackenzie, Phys. Rev. D 48, 2250 (1993).

[12] C. T. H. Davies et al, Phys. Rev. D37 (1988) 1581.

[13] C. T. H. Davies and B. A. Thacker, Nucl. Phys. B405, (1993) 593.

[14] Particle Data Group, Phys. Rev. D50 (1994) 1173.

[15] A. J. Lidsey, PhD thesis, University of Glasgow 1995.

[16] NRQCD collaboration, Proceedings of LAT94, Bielefeld, October 1994, Nucl. Phys. B (Proc. Suppl.) (in press).

[17] See for instance D. H. Perkins, Introduction to High Energy Physics, Third Edition, Addison-Wesley.

[18] S. Collins, Proceedings of LAT94, Bielefeld, October 1994, Nucl. Phys. B (Proc. Suppl.) (in press); A. Ali Khan, ibid.

[19] A. El-Khadra and B. P. Mertens, Proceedings of LAT94, Bielefeld, October 1994, Nucl. Phys. B (Proc. Suppl.) (in press), and reported in review article by J. Shigemitsu, Proceedings of the XXVII International Conference on High Energy Physics, Glasgow 1994, IoP Publishing.

[20] M. Peskin in Dynamics and Spectroscopy at High Energy, Proceedings of the 11th SLAC Summer Institute on Particle Physics, SLAC Report 267.

[21] C. Michael, Phys. Rev. Lett. 56 (1986) 1219.

[22] A. Huntley and C. Michael, Nucl. Phys. B 286 (1987) 211.

[23] G. S. Bali et al, Confining Forces and String Formation from the Lattice, preprint HLRZ-94-41.

[24] A. X. El Khadra, et al, Phys. Rev. Lett. 69 (1992) 729; A. X. El Khadra, Nucl. Phys. (Proc. Suppl.) 34 (1994) 141.

[25] S. Aoki et al, preprint UTHEP-280 (1994), hep-lat 9407015; Nucl. Phys. B. (Proc. Suppl.) 34 (1994) 492. 